\title{
Designing the refractive indices by using magnetic fluids
}

\author{
H. E. Horng ${ }^{\text {a) }}$ \\ Department of Physics, National Taiwan Normal University, Taipei 116, Taiwan and Institute of Electro- \\ optical Science And Technology, National Taiwan Normal University, Taipei 116, Taiwan \\ Chin-Yih Hong \\ Department of Mechanical and Automation Engineering, Da-Yeh University, Chang-Hwa 515, Taiwan
}

S. Y. Yang

Insitutte of Physics, Academia Sinica, Taipei 115, Taiwan

H. C. Yang

Department of Physics, National Taiwan University, Taipei 106, Taiwan

(Received 21 August 2002; accepted 21 February 2003)

\begin{abstract}
Magnetic fluid is a type of colloid consisting of magnetic nanoparticles dispersed in liquid carriers with the aid of surfactants and Brownian motion. Thus, the optical properties of magnetic fluids can be manipulated through the careful selection of magnetic particles and carriers. In this work, we give an example by designing a refractive index by using magnetic fluids composed of various carriers and particles, around 1.465 at $1.557 \mu \mathrm{m}$ wavelength, which plays an important role in optical fiber communication. In addition, we also show how to achieve a desired flexibility in the tunable refractive index with externally varying fields by adopting suitable magnetic fluid films. These results reveal the feasibility of developing index-match or index-tunable devices using magnetic fluids. (C) 2003 American Institute of Physics. [DOI: 10.1063/1.1568147]
\end{abstract}

With the vast development in optoelectronics and communications, research on devices, whose properties can be manipulated by varying the refractive index, has become important because of the tunability and flexibility of these devices. For example, long-period fiber gratings, transmissive devices that couple the copropagation core and cladding modes of the fiber, are very useful for loss filtering and are usually used to flatten the spectra of erbium-doped-fiber amplifiers in optical fiber communication. ${ }^{1}$ The core mode transmission losses of the long-period fiber gratings are expected to be modulated by varying the surrounding refractive index. Stegell et $_{\text {al. }}{ }^{2}$ used several types of liquid media for obtaining various surrounding refractive indices and indicated that the coupling wavelength between the core and the cladding modes shifted to shorter wavelengths as the refractive index of the liquid surrounding the long-period fiber grating increased. Once the refractive index of the liquid medium exceeds that of the cladding, the coupling wavelength increases for higher refractive indices of liquids, and a high coupling efficiency is also achievable. ${ }^{3}$ These results imply that the desired transmission characterization of a long-period fiber grating can be obtained by embedding the fiber grating in a suitable liquid medium.

In addition to long-period fiber gratings, photonic crystals, in which the dielectric function is spatially periodic, causing their optical properties to be dominated by strong diffraction effects, exhibit a strong dependence of the photonic band gap on the index contrast between the microarrayed columns and the interstitial region. ${ }^{4-6}$ For instance, by reducing the index contrast between the semiconductor columns

\footnotetext{
${ }^{\text {a) }}$ Author to whom correspondence should be addressed at: 88, Sec. 4, TingChou Rd., Taipei 116, Taiwan; electronic mail: phyfv001@ scc.ntnu.edu.tw
}

and the liquid media in the interstitial regions, the forbidden band can be shifted to a lower-frequency region. This means that the forbidden band of the photonic crystal can be tunable by using liquid media of various refractive indices for the interstitial region.

At present, the liquid media used for these devices include several kinds of liquids, such as organic solutions, polymers, or liquid crystals. However, due to the limitation of liquid specimens, only a few values, depending on the liquids used, are available for the refractive index. Thus, the optical properties of the devices are restricted. In this work, we reveal that continuous values of the refractive index can be obtained by using magnetic fluids of various concentrations. Furthermore, for a given concentration, the tunability of the refractive index of a magnetic fluid is also verified. The possibility of designing the required tunable refractive index by using magnetic fluids needs more investigation.

The magnetic fluids used here were synthesized from a chemical coprecipitation method. The magnetic fluid was injected into a glass cell with an area of $4 \times 4 \mathrm{~mm}^{2}$ and a depth from tens to hundreds of micrometers. The magnetic fluid film was then covered with a triangle prism made of $\mathrm{ZnSe}$ or other prisms with a high refractive index. Thus, there exists an interface PM between the prism and the magnetic fluid. The critical angle of total reflection at PM was then measured precisely to determine the refractive indices of the magnetic fluid films. ${ }^{7}$ The wavelength of the light investigated was $1.557 \mu \mathrm{m}$. A uniform magnetic field $H$ was provided by a solenoid. To examine the structural patterns in the magnetic fluid films under external fields, an optical microscope and a charge-coupled device camera were adopted to record the images of these patterns.

Since magnetic fluids consist of magnetic particles dispersed in liquid carriers with the aid of surfactants and 


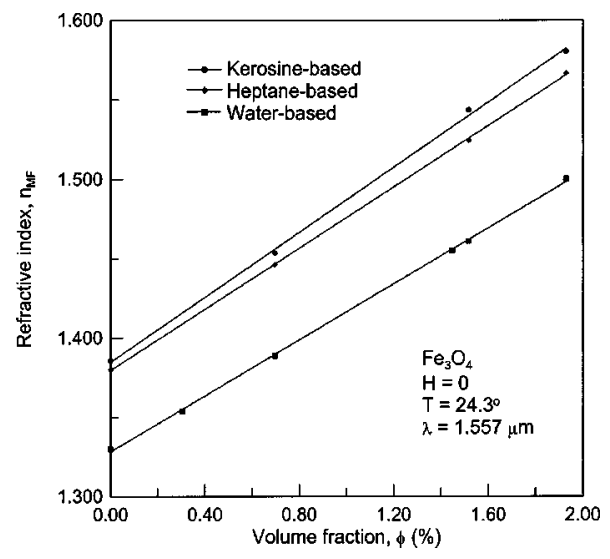

FIG. 1. Refractive index vs concentration of the magnetic fluids under zero field for the carriers of kerosene, heptane, and water using $\mathrm{Fe}_{3} \mathrm{O}_{4}$ as magnetic particles. The wavelength of the light used is $1.557 \mu \mathrm{m}$, and the working temperature is $24.3^{\circ} \mathrm{C}$.

Brownian motion, ${ }^{8}$ the refractive indices of magnetic fluids can be manipulated through the careful selection of magnetic particles and carriers. Before dispersing the magnetic particles into the carrier of the magnetic fluid, the fluid exhibits the intrinsic refractive index of the carrier. With the dispersion of the magnetic particles, the refractive index of magnetic fluid increases from the value corresponding to the carrier linearly and continuously with the particle concentration. Figure 1 shows examples of the concentration dependent refractive indices of the magnetic fluids under zero field. The carriers here are kerosene, heptane, and water, and the magnetic particle is $\mathrm{Fe}_{3} \mathrm{O}_{4}$ with an average diameter around 10 nm. ${ }^{9}$ The surfactant used for both kerosene and heptane is oleic acid, whereas lauric acid is used for water. These three curves in Fig. 1 exhibit a similar behavior except a shift, depending on the used carrier of the magnetic fluid.

According to the results in Fig. 1, the magnetic fluid with a required refractive index, say 1.465 which is close to that of optical fibers, is available by setting the concentration at $0.71 \%$, or at $0.75 \%$ and $1.45 \%$ in terms of volume fraction for the kerosene-, heptane-, and water-based $\mathrm{Fe}_{3} \mathrm{O}_{4}$ magnetic fluids, respectively. Other desired values of the refractive indices for various applications are also possibly obtained by using magnetic fluids with suitable concentrations. It is noted that the highest value of around 1.6 for the refractive index of the magnetic fluid in Fig. 1 is due to the limitations of the experiment. Higher refractive indices of the concentrated magnetic fluids can be probed by using a high-index prism in the experimental setup. ${ }^{7}$

From the past research, we know that the structural pattern formation occurs in the magnetic fluid film under external magnetic fields. ${ }^{10,11}$ This structural formation leads to a change in the refractive index of magnetic fluid films. ${ }^{7}$ This means that we can have index-tunable components by utilizing magnetic fluids. Hence, the structures and the refractive indices of magnetic fluids under external fields are examined.

For the dilute kerosene- and heptane-based magnetic fluids investigated in Fig. 1, the field strength required to achieve the structural formation in the magnetic fluid film is much higher than the maximum field $(375 \mathrm{Oe})$ used available in this work. However, for the dilute water-based $\mathrm{Fe}_{3} \mathrm{O}_{4}$ magnetic fluid films, the field strength responsible for the

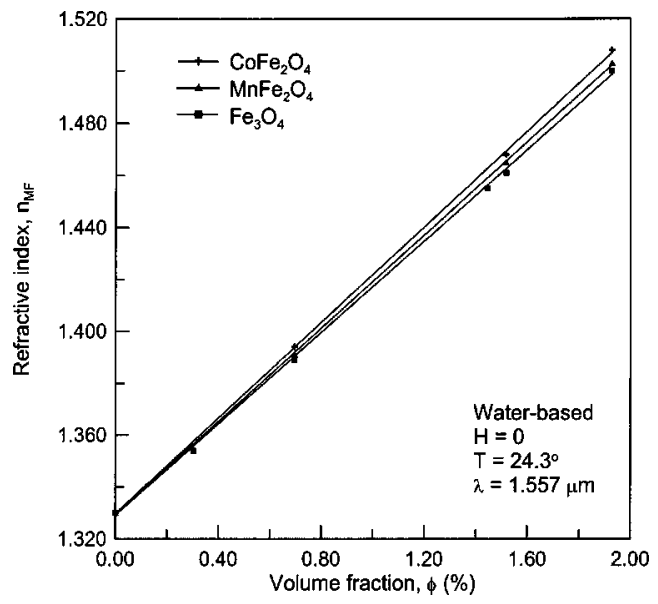

FIG. 2. Concentration-dependent refractive index of the water-based magnetic fluids under zero field. The magnetic particles investigated here are $\mathrm{CoFe}_{2} \mathrm{O}_{4}, \mathrm{MnFe}_{2} \mathrm{O}_{4}$, and $\mathrm{Fe}_{3} \mathrm{O}_{4}$. The wavelength of the light used is 1.557 $\mu \mathrm{m}$, and the temperature is $24.3^{\circ} \mathrm{C}$.

structural formation is only $20-30$ Oe. Therefore, from the application point of view, the water-based magnetic fluids seem the most promising candidate. Hereafter, we investigate the refractive index of water-based magnetic fluid films to illustrate the designing principle for the tunable refractive index.

Figure 2 plots the zero-field refractive index as a function of the concentrations of the magnetic fluids consisting of various kinds of magnetic particles like $\mathrm{CoFe}_{2} \mathrm{O}_{4}$, $\mathrm{MnFe}_{2} \mathrm{O}_{4}$, and $\mathrm{Fe}_{3} \mathrm{O}_{4}$. Slight deviations among these three curves were observed. The three kinds of water-based magnetic fluid films showed two different types of behavior upon applying a magnetic field. For the $\mathrm{CoFe}_{2} \mathrm{O}_{4}$, owing to the high solubility of surfactant-coated $\mathrm{CoFe}_{2} \mathrm{O}_{4}$ in water, no structural formation occurred in the film even below 375 Oe. Consequently, the refractive index of the water-based $\mathrm{CoFe}_{2} \mathrm{O}_{4}$ magnetic fluid remained constant for the field strength range provided in this work. Whereas for $\mathrm{MnFe}_{2} \mathrm{O}_{4}$ and $\mathrm{Fe}_{3} \mathrm{O}_{4}$, the structural patterns in the magnetic fluid films obviously change under external magnetic fields. Typical structural evolutions in the $11.8 \mu \mathrm{m}$ thick magnetic fluid films with the magnetic field are shown in Figs. 3(a) and 3(b) for $\mathrm{MnFe}_{2} \mathrm{O}_{4}$ and $\mathrm{Fe}_{3} \mathrm{O}_{4}$, respectively. The magnetic fields were applied normal to the film. It is clear that the magnetic columns, agglomerated by magnetic particles, were formed in the film under fields. The corresponding qualitative analysis for the area ratio, $A_{\mathrm{col}} / A$, occupied by the columns as a function of the field strength, is shown in Fig. 4. The observed structural formation implies that a magnetically tunable refractive index is achievable by using magnetic fluid films, as shown in Fig. 5.

For the water-based $\mathrm{Fe}_{3} \mathrm{O}_{4}$ magnetic fluid film of certain thickness, say $11.8 \mu \mathrm{m}$, the refractive index starts to increase from 1.462 as the field is higher than 25 Oe and then almost becomes saturated (1.466) when the field reaches 200 Oe. Thus, the sensitivity in the magnetically tunable refractive index of the water-based $11.8 \mu \mathrm{m}$ thick $\mathrm{Fe}_{3} \mathrm{O}_{4}$ magnetic fluid film of is about $0.000023 \mathrm{Oe}^{-1}$. Also, the results imply that the refractive index of the magnetic fluid film can be finely tuned by controlling the field strength. The water-based magnetic fluid film with $\mathrm{MnFe}_{2} \mathrm{O}_{4}$ also behaves in a similar way. 
(a1) $\mathrm{H}=0$

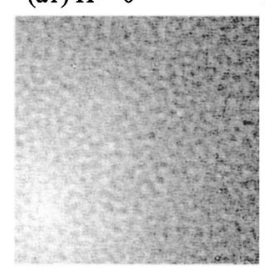

(a2) $\mathrm{H}=150 \mathrm{Oe}$

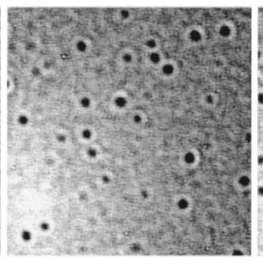

(b1) $\mathrm{H}=0$
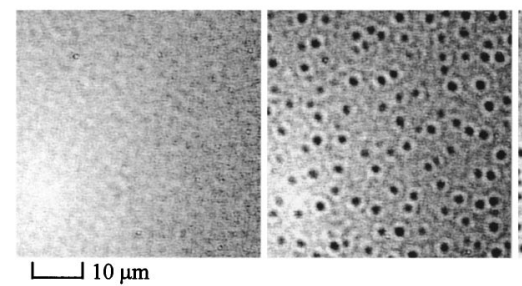

(b3) $240 \mathrm{Oe}$

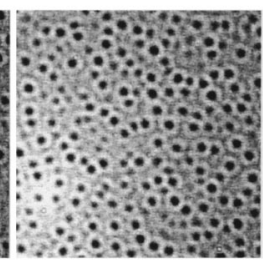

(b2) $\mathrm{H}=150 \mathrm{Oe}$

FIG. 3. Images of the structural patterns under various magnetic fields inside the water-based magnetic fluid films with particles of (a) $\mathrm{Fe}_{3} \mathrm{O}_{4}$ and (b) $\mathrm{MnFe}_{2} \mathrm{O}_{4}$. The film thickness is $11.8 \mu \mathrm{m}$ and the magnetic field is applied normal to the film. The temperature is $24.3^{\circ} \mathrm{C}$. The dark spots denote the cross sections of the magnetic columns agglomerated by particles.

If a larger or a steeper variation in the refractive index with the field is needed, the magnetic fluid film with a larger thickness is suggested. The field dependent refractive indices of thicker (200 $\mu$ m thick) water-based $\mathrm{Fe}_{3} \mathrm{O}_{4}$ and $\mathrm{MnFe}_{2} \mathrm{O}_{4}$ magnetic fluid films are plotted in Fig. 5. The sensitivity in the variation of refractive index due to the varying field is about $0.0085 \mathrm{Oe}^{-1}$. Thus, the required sensitivity in the magnetically tunable refractive index can be obtained by adequately selecting the thickness of the magnetic fluid film. On the other hand, the variation in the range of tunable refractive index shown in Fig. 5 can be shifted to a higher/ lower value by adopting a more concentrated/dilute magnetic fluid. It is worth noting that the tunable refractive indices of magnetic fluids are useful for developing dynamic electrooptical devices that switch on and off alternatively, such as optical switches or modulators. Thus, the magnetic aging effect, which plays an important role in the optical properties

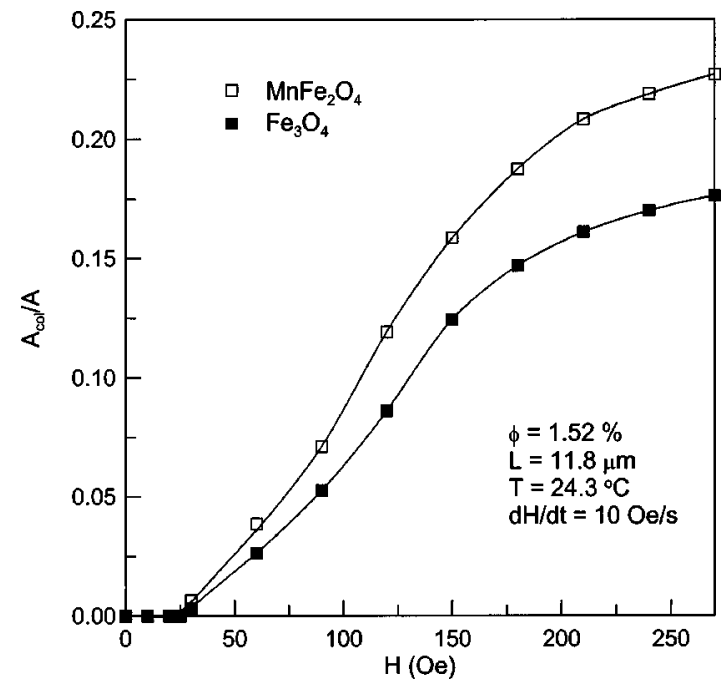

FIG. 4. Area ratio $A_{\text {col }} / A$ as a function of the magnetic field strength for the water-based magnetic fluid films with $\mathrm{MnFe}_{2} \mathrm{O}_{4}$ and $\mathrm{Fe}_{3} \mathrm{O}_{4}$ particles at $24.3^{\circ} \mathrm{C}$.

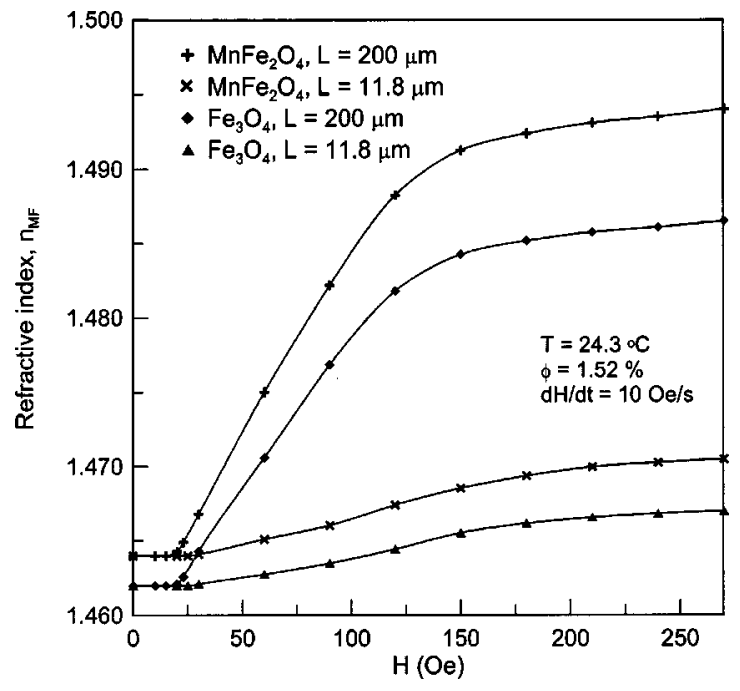

FIG. 5. Magnetically modulated refractive index of the water-based magnetic fluid films of various thickness and magnetic particles for the wavelength of $1.557 \mu \mathrm{m}$ at $24.3^{\circ} \mathrm{C}$. A sharper change in the refractive index with the field strength is resulted for a thicker magnetic fluid film.

of magnetic fluids in a static magnetic field for a period of time, ${ }^{12,13}$ on the field-dependent refractive index of magnetic fluids, can be ignored. If one needs a fixed value of the refractive index in practical applications, the magnetic fluid with an adequate concentration under zero field can be applied.

In conclusion, we have illustrated the designing principle for the tunable refractive index by using magnetic fluids. One can select the adequately concentrated magnetic fluid with the refractive index around the desired value, which depends on the applications, then finely tune the refractive index by applying a magnetic field. The sensitivity in the field-dependent refractive index can also be adjusted by controlling the film thickness.

This work is supported by National Science Council of ROC under Grant Nos. NSC90-2112-M-003-020, NSC 902112-E-212-031, and Grant No. 91-N-FA01-2-4-2.

${ }^{1}$ A. M. Vengasarkar, P. J. Lemaire, J. B. Judkins, V. Bhatia, T. Erdogan, and J. E. Sipe, J. Lightwave Technol. 14, 58 (1996).

${ }^{2}$ D. B. Stegall and T. Erdogan, IEEE Photonics Technol. Lett. 11, 343(1999).

${ }^{3}$ O. Duhem, J.-F. Henninot, M. Warenghem, and M. Douay, Appl. Opt. 37, 7223 (1998).

${ }^{4}$ H.-B. Lin, R. J. Tonucci, and A. J. Campillo, Appl. Phys. Lett. 68, 2927 (1996).

${ }^{5}$ J. F. Bertone, P. Jiang, K. S. Hwang, D. M. Mittleman, and V. L. Colvin, Phys. Rev. Lett. 83, 300 (1999).

${ }^{6}$ K. Yoshino, S. Satoh, Y. Shimoda, and Y. Kawagishi, Jpn. J. Appl. Phys., Part 2 38, L961 (1999).

${ }^{7}$ S. Y. Yang, Y. F. Chen, H. E. Horng, Chin-Yih Hong, W. S. Tse, and H. C. Yang, Appl. Phys. Lett. 81, 4931 (2002).

${ }^{8}$ R. E. Rosensweig, Ferrohydrodynamics (Cambridge University Press, New York, 1985).

${ }^{9}$ H. E. Horng, I. J. Jang, K. L. Kung, Y. D. Yao, H. C. Yang, and C.-Y. Hong, Czech. J. Phys. 46, S4, 2023 (1996)

${ }^{10}$ C.-Y. Hong, I. J. Jang, H. E. Horng, C. J. Hsu, Y. D. Yao, and H. C. Yang, J. Appl. Phys. 81, 4275 (1997).

${ }^{11}$ C.-Y. Hong, C. A. Chen, C.-H. Chen, H. E. Horng, S. Y. Yang, and H. C. Yang, Appl. Phys. Lett. 79, 2360 (2001).

${ }^{12}$ K. Skeff Neto, A. F. Bakuzis, A. R. Pereira, and P. C Morais, J. Magn. Magn. Mater. 226, 1893 (2001).

${ }^{13}$ K. Skeff Neto, A. F. Bakuzis, P. C. Morais, A. R. Pereira, R. B. Azevedo, L. M. Lacava, and Z. G. M. Lacava, J. Appl. Phys. 89, 3362 (2001). 
Applied Physics Letters is copyrighted by the American Institute of Physics (AIP). Redistribution of journal material is subject to the AIP online journal license and/or AIP copyright. For more information, see http:/ojps.aip.org/aplo/aplcr.jsp

Copyright of Applied Physics Letters is the property of American Institute of Physics and its content may not be copied or emailed to multiple sites or posted to a listserv without the copyright holder's express written permission. However, users may print, download, or email articles for individual use. 